\title{
AN INEQUALITY FOR PROBABILITY DENSITY FUNCTIONS ARISING FROM A DISTINGUISHABILITY PROBLEM
}

\author{
BORIS GULJAŠ' , C. E. M. PEARCE ${ }^{2}$ and JOSIP PEČARIĆ ${ }^{3}$
}

(Received 2 August 1995)

\begin{abstract}
An integral inequality is established involving a probability density function on the real line and its first two derivatives. This generalizes an earlier result of Sato and Watari. If $f$ denotes the probability density function concerned, the inequality we prove is that$$
\int_{-\infty}^{+\infty} \frac{\left[f^{\prime}(x)^{2}\right]^{\gamma \alpha}}{[f(x)]^{\gamma(\beta+1)-1}} d x \leq\left(\frac{2 \alpha-1}{\beta-1}\right)^{\gamma \alpha}\left(\int_{-\infty}^{+\infty} \frac{\left|f^{\prime \prime}(x)\right|^{\alpha-1}}{[f(x)]^{\beta-\alpha}} d x\right)^{\gamma}
$$

under the conditions $\beta>\alpha>1$ and $1 /(\beta+1)<\gamma \leq 1$.
\end{abstract}

\section{Introduction}

In this article we establish a general integral inequality involving a probability density and its first two derivatives (in the distributional sense). Integrals of the sort involved can arise in probabilistic extremal problems via the calculus of variations and optimal control theory and our result has an interest for such applications.

The genesis of the present ideas lies in a striking distinguishability problem whose roots go back half a century to a paper of Kakutani [2]. Suppose $X=\left(X_{i}\right)_{1}^{\infty}$ is a sequence of independent and identically distributed random variables and $a=\left(a_{i}\right)_{1}^{\infty}$ an associated numerical sequence, $a_{i}$ representing the error in centering $X_{i}$. When are the sample paths $X$ and $X+a$ distinguishable?

A key concept to unlock this question is that of finite information. We say that $X$ has finite information if the common distribution of the $X_{i}$ has an almost surely positive and (locally) absolutely continuous density function $f$ satisfying

$$
I_{1}(f) \equiv \int_{-\infty}^{\infty}\left[f^{\prime}(x)\right]^{2} / f(x) d x<\infty
$$

\footnotetext{
'Department of Mathematics, University of Zagreb, Bijenička c. 30, 41000 Zagreb, Croatia ${ }^{2}$ Department of Applied Mathematics, The University of Adelaide, Adelaide SA 5005, Australia ${ }^{3}$ Faculty of Textile Technology, University of Zagreb, Pierottijeva 6, 41000 Zagreb, Croatia (c) Australian Mathematical Society, 1998, Serial-fee code 0334-2700/98
} 
where $f^{\prime}$ is the derivative of $f$ in the distributional sense. For $X$ with finite information we can distinguish if and only if $\sum a_{i}^{2}=\infty$. These results were established by Shepp [6], who made use of the machinery of the Hilbert space $L_{2}$ of squareintegrable functions and of some work of Kakutani on the equivalence of infinite product measures. This introduces the stronger concept of total indistinguishability, which signifies in physical terms that for every observed sequence, there is doubt as to whether it came from $X$ or from $X+a$. A necessary and sufficient condition for total indistinguishability is that the infinite product measures $\mu_{X}$ and $\mu_{X+a}$ induced by $X$ and $X+a$ be mutually absolutely continuous or equivalent, denoted by $\mu_{X} \sim \mu_{X+a}$.

Subsequently there was exploration of the more general question where $a$ is replaced by an identically and independently distributed sequence $Y=\left(Y_{i}\right)_{1}^{\infty}$ of symmetric random variables. Kitada and Sato [3] have given sufficient conditions for distinguishability under the requirement

$$
I_{2}(f) \equiv \int_{-\infty}^{\infty}\left[f^{\prime \prime}(x)\right]^{2} / f(x) d x<\infty .
$$

They proved also that (1.2) implies (1.1) if $f$ is monotone for large $|x|$.

More recently Sato and Watari [5] established that

$$
I_{1}(f) \leq \frac{3}{2}\left[I_{2}(f)\right]^{3 / 2},
$$

showing that (1.2) implies (1.1) quite generally. They derive as an application that if (1.2) holds and the distributions of $Y$ are symmetric with $Y \in \ell_{4}$ almost surely, then $X+Y$ and $X$ induce mutually absolutely continuous probability measures and so are totally indistinguishable. Here $\ell_{\alpha}$ (for $\alpha>1$ ) denotes the space of all random sequences such that $\sum_{k=1}^{\infty} Y_{k}^{\alpha}<\infty$ a.s. The condition $Y \in \ell_{4}$ a.s. had arisen in early work by Rozanov [4] and Fernique [1] as a necessary and sufficient condition for equivalence of the measures on sequence space in the case when $X, Y$ are centred Gaussian.

In the present paper we provide a generalization of (1.3). To be specific, suppose that $f$ is an a.s. positive density function and write

$$
I_{\gamma, \alpha, \beta}=\int_{-\infty}^{+\infty} \frac{\left[f^{\prime}(x)^{2}\right]^{\gamma \alpha}}{[f(x)]^{\gamma(\beta+1)-1}} d x, \quad J_{\alpha, \beta}=\int_{-\infty}^{+\infty} \frac{\left|f^{\prime \prime}(x)\right|^{\alpha}}{[f(x)]^{\beta-\alpha}} d x
$$

In Section 2 we establish the following result.

THEOREM 1. If $f$ is such that $J_{\alpha, \beta}<\infty$ for some $\beta>\alpha>1$, then

$$
I_{\gamma, \alpha . \beta} \leq\left(\frac{2 \alpha-1}{\beta-1}\right)^{\gamma \alpha} J_{\alpha . \beta}^{\gamma}
$$

for $1 /(\beta+1)<\gamma \leq 1$. 
This includes the inequalities of Sato and Watari [5] as the special cases $\alpha=2$, $\beta=3, \gamma=1$ and $\gamma=1 / 2$.

\section{Proof of Theorem 1}

The proof of Theorem 1 is based on the following lemma.

LEMMA 1. Let $[a, b],(-\infty \leq a<b \leq+\infty)$ be a closed interval and $h$ a nonnegative continuously differentiable function on $[a, b]$. Suppose the derivative $h^{\prime}$ is an absolutely continuous non-vanishing function on $(a, b), h^{\prime}(a+)=h^{\prime}(b-)=0$, and $\int_{a}^{b}\left|h^{\prime \prime}(x)\right|^{\alpha} /[h(x)]^{\beta-\alpha} d x<\infty$ for the second derivative $h^{\prime \prime}$ when $\beta>\alpha>0$. Then

$$
\int_{a}^{b} \frac{\left[h^{\prime}(x)^{2}\right]^{\alpha}}{[h(x)]^{\beta}} d x=\frac{2 \alpha-1}{\beta-1} \int_{a}^{b} \frac{\left[h^{\prime}(x)^{2}\right]^{\alpha-1} h^{\prime \prime}(x)}{[h(x)]^{\beta-1}} d x,
$$

$\int_{a}^{b} \frac{\left[h^{\prime}(x)^{2}\right]^{\gamma \alpha}}{[h(x)]^{\gamma(\beta+1)-1}} d x \leq\left(\frac{2 \alpha-1}{\beta-1}\right)^{\gamma \alpha}\left(\int_{a}^{b} h(x) d x\right)^{1-\gamma}\left(\int_{a}^{b} \frac{\left|h^{\prime \prime}(x)\right|^{\alpha}}{[h(x)]^{\beta-\alpha}} d x\right)^{\gamma}$

for $1 /(\beta+1)<\gamma \leq 1$.

PROOF OF LemMA 1. For $\eta>0$ we define $h_{\eta}(x)=h(x)+\eta$ as in [5]. For every $\beta-1>\varepsilon>0$, since $h^{\prime}(a+)=h^{\prime}(b-)=0$, we can choose a closed interval $\left[a^{\prime}, b^{\prime}\right]$ contained in $(a, b)$ such that

$$
\left|\left[\frac{\left[h^{\prime}(x)^{2}\right]^{\alpha}}{\left[h_{\eta}(x)\right]^{\beta-1}}\right]_{a^{\prime}}^{b^{\prime}}\right| \leq \varepsilon \int_{a^{\prime}}^{b^{\prime}} \frac{\left[h^{\prime}(x)^{2}\right]^{\alpha}}{\left[h_{\eta}(x)\right]^{\beta}} d x .
$$

Further we have

$$
\begin{aligned}
\int_{a^{\prime}}^{b^{\prime}} \frac{\left[h^{\prime}(x)^{2}\right]^{\alpha-1} h^{\prime \prime}(x)}{\left[h_{\eta}(x)\right]^{\beta-1}} d x= & \int_{a^{\prime}}^{b^{\prime}} \frac{\left[h^{\prime}(x)^{2}\right]^{\alpha}}{\left[h_{\eta}(x)\right]^{\beta-1}} \frac{h^{\prime \prime}(x)}{\left[h^{\prime}(x)^{2}\right]} d x \\
=- & -\left[\frac{\left[h^{\prime}(x)\right]^{(2 \alpha-1)}}{\left[h_{\eta}(x)\right]^{\beta-1}}\right]_{a^{\prime}}^{b^{\prime}}+2 \alpha \int_{a^{\prime}}^{b^{\prime}} \frac{\left[h^{\prime}(x)^{2}\right]^{\alpha-1} h^{\prime \prime}(x)}{\left[h_{\eta}(x)\right]^{\beta-1}} d x \\
& -\beta \int_{a^{\prime}}^{b^{\prime}} \frac{\left[h^{\prime}(x)^{2}\right]^{\alpha}}{\left[h_{\eta}(x)\right]^{\beta}} d x,
\end{aligned}
$$

that is,

$$
\int_{a^{\prime}}^{b^{\prime}} \frac{\left[h^{\prime}(x)^{2}\right]^{\alpha}}{\left[h_{\eta}(x)\right]^{\beta}} d x=\frac{2 \alpha-1}{\beta-1} \int_{a^{\prime}}^{b^{\prime}} \frac{\left[h^{\prime}(x)^{2}\right]^{\alpha-1} h^{\prime \prime}(x)}{\left[h_{\eta}(x)\right]^{\beta-1}} d x-\frac{1}{\beta-1}\left[\frac{\left[h^{\prime}(x)\right]^{(2 \alpha-1)}}{\left[h_{\eta}(x)\right]^{\beta-1}}\right]_{a^{\prime}}^{b^{\prime}} .
$$


From (2.3) and Hölder's inequality we have

$$
\begin{aligned}
\int_{a^{\prime}}^{b^{\prime}} \frac{\left[h^{\prime}(x)^{2}\right]^{\alpha}}{\left[h_{\eta}(x)\right]^{\beta}} d x \leq & \frac{2 \alpha-1}{\beta-1-\varepsilon} \int_{a^{\prime}}^{b^{\prime}} \frac{\left[h^{\prime}(x)^{2}\right]^{\alpha-1} h^{\prime \prime}(x)}{\left[h_{\eta}(x)\right]^{\beta-1}} d x \\
\leq & \frac{2 \alpha-1}{\beta-1-\varepsilon}\left[\int_{a^{\prime}}^{b^{\prime}}\left(\frac{\left[h^{\prime}(x)^{2}\right]^{\alpha-1}}{\left[h_{\eta}(x)\right]^{(\alpha-1) \beta / \alpha}}\right)^{\alpha /(\alpha-1)} d x\right]^{1-1 / \alpha} \\
& \times\left[\int_{a^{\prime}}^{b^{\prime}}\left(\frac{\left|h^{\prime \prime}(x)\right|}{\left[h_{\eta}(x)\right]^{(\beta-\alpha) / \alpha}}\right)^{\alpha} d x\right]^{1 / \alpha} \\
\leq & \frac{2 \alpha-1}{\beta-1-\varepsilon}\left(\int_{a^{\prime}}^{b^{\prime}} \frac{\left[h^{\prime}(x)^{2}\right]^{\alpha}}{\left[h_{\eta}(x)\right]^{\beta}} d x\right)^{1-1 / \alpha} \\
& \times\left(\int_{a^{\prime}}^{b^{\prime}} \frac{\left|h^{\prime \prime}(x)\right|^{\alpha}}{\left[h_{\eta}(x)\right]^{\beta-\alpha}} d x\right)^{1 / \alpha}
\end{aligned}
$$

From the last inequality we have

$$
\begin{aligned}
\int_{a^{\prime}}^{b^{\prime}} \frac{\left[h^{\prime}(x)^{2}\right]^{\alpha}}{\left[h_{\eta}(x)\right]^{\beta}} d x & \leq\left(\frac{2 \alpha-1}{\beta-1-\varepsilon}\right)^{\alpha} \int_{a^{\prime}}^{b^{\prime}} \frac{\left|h^{\prime \prime}(x)\right|^{\alpha}}{\left[h_{\eta}(x)\right]^{\beta-\alpha}} d x \\
& \leq\left(\frac{2 \alpha-1}{\beta-1-\varepsilon}\right)^{\alpha} \int_{a}^{b} \frac{\left|h^{\prime \prime}(x)\right|^{\alpha}}{[h(x)]^{\beta-\alpha}} d x \\
& <\infty
\end{aligned}
$$

On taking $\varepsilon \rightarrow 0$ together with $a^{\prime} \searrow a$ and $b^{\prime} \nearrow b$ we have

$$
\begin{aligned}
& \int_{a}^{b} \frac{\left[h^{\prime}(x)^{2}\right]^{\alpha}}{\left[h_{\eta}(x)\right]^{\beta}} d x=\frac{2 \alpha-1}{\beta-1} \int_{a}^{b} \frac{\left[h^{\prime}(x)^{2}\right]^{\alpha-1} h^{\prime \prime}(x)}{\left[h_{\eta}(x)\right]^{\beta-1}} d x, \\
& \int_{a}^{b} \frac{\left[h^{\prime}(x)^{2}\right]^{\alpha}}{\left[h_{\eta}(x)\right]^{\beta}} d x \leq\left(\frac{2 \alpha-1}{\beta-1}\right)^{\alpha} \int_{a}^{b} \frac{\left|h^{\prime \prime}(x)\right|^{\alpha}}{\left[h_{\eta}(x)\right]^{\beta-\alpha}} d x .
\end{aligned}
$$

Relations (2.1) and (2.2) for $\gamma=1$ follow from (2.4) and (2.5) on letting $\eta \searrow 0$.

If $1 /(\beta+1)<\gamma<1,(2.2)$ follows from Hölder's inequality and (2.2) for $\gamma=1$, that is,

$$
\begin{aligned}
\int_{a}^{b} \frac{\left[h^{\prime}(x)^{2}\right]^{\gamma \alpha}}{[h(x)]^{\gamma(\beta+1)-1}} d x & =\int_{a}^{b} \frac{\left[h^{\prime}(x)^{2}\right]^{\gamma \alpha}}{[h(x)]^{\gamma \beta}}[h(x)]^{1-\gamma} d x \\
& \leq\left(\int_{a}^{b} h(x) d x\right)^{1-\gamma}\left(\int_{a}^{b} \frac{\left[h^{\prime}(x)^{2}\right]^{\alpha}}{[h(x)]^{\beta}} d x\right)^{\gamma} \\
& \leq\left(\frac{2 \alpha-1}{\beta-1}\right)^{\gamma \alpha}\left(\int_{a}^{b} h(x) d x\right)^{1-\gamma}\left(\int_{a}^{b} \frac{\left|h^{\prime \prime}(x)\right|^{\alpha}}{[h(x)]^{\beta-\alpha}} d x\right)^{\gamma} .
\end{aligned}
$$


Proof OF TheOrem 1. As in [5], the continuity of $f^{\prime}$ implies that $R \backslash\left\{x \mid f^{\prime}(x)=0\right\}$ is the union of at most a countable number of mutually disjoint open intervals $\left(a_{n}, b_{n}\right)$ such that $f$ satisfies the hypotheses of Lemma 1 on each closed interval $\left[a_{n}, b_{n}\right]$. By applying (2.2) and Hölder's inequality we have

$$
\begin{aligned}
\int_{-\infty}^{+\infty} \frac{\left[f^{\prime}(x)^{2}\right]^{\gamma \alpha}}{[f(x)]^{\gamma(\beta+1)-1}} d x= & \sum_{n} \int_{a_{n}}^{b_{n}} \frac{\left[f^{\prime}(x)^{2}\right]^{\gamma \alpha}}{[f(x)]^{\gamma(\beta+1)-1}} d x \\
\leq & \left(\frac{2 \alpha-1}{\beta-1}\right)^{\gamma \alpha} \sum_{n}\left(\int_{a_{n}}^{b_{n}} f(x) d x\right)^{1-\gamma} \\
& \times\left(\int_{a_{n}}^{b_{n}} \frac{\left|f^{\prime \prime}(x)\right|^{\alpha}}{[f(x)]^{\beta-\alpha}} d x\right)^{\gamma} \\
\leq & \left(\frac{2 \alpha-1}{\beta-1}\right)^{\gamma \alpha}\left(\sum_{n} \int_{a_{n}}^{b_{n}} f(x) d x\right)^{1-\gamma} \\
& \times\left(\sum_{n} \int_{a_{n}}^{b_{n}} \frac{\left|f^{\prime \prime}(x)\right|^{\alpha}}{[f(x)]^{\beta-\alpha}} d x\right)^{\gamma} \\
\leq & \left(\frac{2 \alpha-1}{\beta-1}\right)^{\gamma \alpha}\left(\int_{-\infty}^{+\infty} \frac{\left|f^{\prime \prime}(x)\right|^{\alpha}}{[f(x)]^{\beta-\alpha}} d x\right)^{\gamma} .
\end{aligned}
$$

\section{References}

[1] X. Fernique, "Régularité des trajectoires des fonctions aléatoires gaussiennes", in Lecture Notes in Math. 480 (Springer-Verlag, New York, 1974), pp. 1-96.

[2] S. Kakutani, "On equivalence of infinite product measures", Ann. of Math. 49 (1948) 214-224.

[3] K. Kitada and H. Sato, "On the absolute continuity of infinite product measure and its convolution", Probab. Theory Related Fields 81 (1989) 609-627.

[4] Yu. A. Rozanov, "On the density of one Gaussian measure with respect to another", Theory Probab. Appl. 7 (1962) 82-87.

[5] H. Sato and C. Watari, "Some integral inequalities and absolute continuity of a symmetric random translation", J. Functional Anal. 114 (1993) 257-266.

[6] L. A. Shepp, "Distinguishing a sequence of random variables from a translate of itself", Ann. Math. Statist. 36 (1965) 1107-1112. 